$\mathrm{p}<.02 ;$ form $\chi^{2}=50.66, \mathrm{df}=4, \mathrm{p}<.001 ;$ orientation, $\chi^{2}=21, \mathrm{~d} f=4, \mathrm{p}<.001$ ). Except for the nonsignificant chi square for Group $N$ for form, the first presentation analyses yield the same findings as the analyses on overall data.

Since direct comparisons between Groups $N$ and $O$ cannot be made on absolute number of responses due to the procedures employed, each S's total number of responses made to $S+$ for each dimension was converted into percentages. On these percentage scores, three Mann-Whitney $\mathbf{U}$ tests were performed, comparing Groups $\mathrm{N}$ and $\mathrm{O}$ for each dimension. These analyses revealed significant differences between the two groups for form $\left(U=22.5, \quad n_{1}=15\right.$, $\left.\mathrm{n}_{2}=15, \mathrm{p}<.001\right)$ and orientation $\left(\mathrm{U}=39.8, \mathrm{n}_{1}=15, \mathrm{n}_{2}=15, \mathrm{p}<.001\right)$, but not for color $\left(U=1.00, n_{1}=15, n_{2}=15\right.$, $\mathrm{p}>.05$ ).

Calculations of the percentage of $\mathrm{S}+$ responses to each dimension for Group $\mathrm{O}$ on overall data indicated that $88 \%$ of the responses to the form dimension were to St, while only $65 \%$ of the responses to the orientation dimension and $38 \%$ of the color responses were to $\mathbf{S}+$. Thus, Group O's group data appears to indicate that in addition to learning the three attributes, $S$ hierarchically orders the attributes with form as dominant. In order to determine if this group distribution characterized individual behavior, the correspondence between the group and individual functions was analyzed. Table 1 presents the distribution of test responses to each dimension for each $S$ in Group $O$. An attribute will be considered learned by $S$ if $S$ made at least three responses to the $S+$ stimulus within each dimensional array. Applying this criterion, inspection of Table 1 indicates the following: (1) Three Ss learned three attributes, five Ss learned two attributes, and seven Ss learned only one attribute; and (2) for those Ss (Nos. 1 through 8) who learned two or three attributes, there is little indication (however, see S 8) of differences in response strength between learned attributes.

In order to determine how the individual data contributed to group functions, the number of Ss learning a particular attribute was calculated. As may be seen in Table 1, 14 Ss learned the form of S+, 8 Ss learned the orientation of $\mathrm{S}+$, and $4 \mathrm{Ss}$ learned the color of $\mathrm{St}$. In summary, inspection of individual data revealed that (1) individual Ss learn one, two, or three attributes of S+ (in contrast to group data analyses, which suggested that all Ss learned three attributes) and (2) the group functions, which indicated that $88 \%$ of the test responses to the form dimension were to
$\mathrm{S}+, 65 \%$ of the orientation responses were to $S+$, and $38 \%$ of the color responses were to $S+$, may be accounted for by the fact that $93 \%$ of the Ss learned the form of St, whereas $53 \%$ of the Ss leamed the orientation, and only $27 \%$ of the Ss learned the color.

\section{DISCUSSION}

The primary purpose of this experiment was to investigate the hypothesis that flat generalization patterns for line orientation indicate that the orientation attribute of the St training complex does not control test behavior but need not indicate that the orientation attribute was not learned. Thus, the hypothesis distinguished between "what is learned" and what is indicated under limited test conditions. Results from the approximated successive testing procedure (Group N) indicated that the orientation attribute of $\mathrm{S}+$ did not control test behavior. However, the results from Group $\mathrm{O}$ indicated that the orientation attribute was learned by at least $53 \%$ of the Ss. Thus, flat line-orientation patterns obtained under successive test conditions does not indicate S's failure to learn the orientation attribute.

The second purpose of this investigation was to determine whether or not an untested-for $\mathrm{S}+$ attribute [its form (a line) or its color (black)] controls responding under conditions which approximate successive testing (Group N). The results from Group N provided little evidence that the form and color attributes were learned. These results allow no conclusions as to the controlling stimulus attribute (or attributes) in this test condition. The only reasonable conclusion is that some still untested-for attribute controls responding. The results from Group $\mathrm{O}$ indicated that $93 \%$ of Ss learned the form of $S+$, whereas $27 \%$ of Ss learned the color. This result indicated that the form attribute and color attribute of the S+, although learned by the $S$ s, did not exert response control under approximated successive testing.

With the assumption that the $O$ procedure had been maximally sensitive in detecting that learning had occurred on the dimensions tested, the results of the present experiment suggest the operation of activation and selection (utilization) mechanisms. More specifically, the results suggest that (1) during training the $\mathrm{S}+$ complex activates, within individuals, one, two, or three attribute mediators; and that (2) test procedures which "force" S (Group 0) to select one stimulus from a dimensional array permits $S$ to select the appropriate mediator from those activated ("learned") during training, to control test behavior.

\section{REFERENCES}

KALISH, H. 1. Stimulus generalization. In M. H. Marx (Ed.), Learning: Processes. London: Macmillan, 1969. Pp. 207-297.

LANDAU, J. S. Postdiscrimination generalization in human subjects of two different ages. Journal of Experimental Psychology, 1968a, 76, 656-663.

LANDAU, J. S. Line-orientation generalization in children and adults as a function of the number of training trials. Psychonomic Science, 1968b, 13, 219-220.

LANDAU, J. S. Size generalization in children as a function of test method and age. Psychonomic Science, 1969, 16, 57-58.

LASHLEY, K. S., \& WADE, M. The Pavlovian theory of generalization. Psychological Review, 1946, 53, 72-87.

PROKASY, W. J., \& HALL, J. F. Primary stimulus generalization. Psychological Review, 1963, 70, 310-322.

SIEGEL, S. Nonparametric statistics for the behavioral sciences. New York: McGraw-Hill, 1956.

1. We would like to thank Mrs. Jacobs of the Corner School Nursery and Rabbi Gellman of the Forest Park Day Camp for their kind cooperation in providing subjects.

\title{
The effect of anchoring upon pain threshold
}

\author{
DIANA R. HASLAM \\ University of Bristol, Bristol 8, England
}

An experiment designed to measure the effect of anchoring upon the heat-pain threshold is reported. The anchor stimulus, which took the form of repeated application of a low-intensity stimulus prior to the assessment of pain threshold, was found to have a significant effect. The conclusion was drawn that the low threshold values found when a small stimulus interval is used in the assessment of heat-pain threshold by the limiting method can probably be attributed to this effect.

In two previous experiments (Haslam, 1965; Haslam \& Thomas, 1967), it was found that when heat-pain threshold was assessed by the limiting method the size of the increment in successive values of the variable stimulus had a considerable effect upon the results. When the increment was as small as $8 \mathrm{mc} / \mathrm{sec} / \mathrm{cm}^{2}$, pain threshold was significantly lower than when the variable stimulus was increased in steps twice or four times as large as this. In an attempt to explain this finding, two 
Table 1

Mean Pain Threshold and Range for the Experimental and Control Conditions Referred to in the Text

\begin{tabular}{lcc}
\hline Conditions & $\begin{array}{c}\text { Mean Pain Threshold } \\
\text { in mc/sec/cm }\end{array}$ & Range \\
\hline Experimental & 209 & $124-264$ \\
Control & 240 & $176-320$ \\
\hline
\end{tabular}

hypotheses, the "tolerance-limit hypothesis" and the "suggestibility hypothesis," were tested in a further experiment (Haslam, 1968). Neither of these hypotheses was confirmed, and so some other explanation of the results was sought. It was argued that an anchoring effect, similar to that described by Helson (1947), might account for the low pain threshold values found when a small stimulus interval is used. An implication of Helson's theory is that the effect of an anchor stimulus upon adaptation level (AL) will increase as some function of the frequency of its application. It was therefore decided to introduce into the usual procedure for pain-threshold assessment an anchor in the form of repeated application of the starting-point stimulus. It was predicted that pain thresholds would be lower under these conditions than under conditions in which there was no such anchor. There were two sessions, and each $S$ served as his own control.

\section{METHOD}

Pain threshold was assessed by means of a modified Hardy.Wolff (1952) radiant-heat apparatus. Details of this and the procedure used are given elsewhere (Haslam, 1965).

The energy level of the starting-point stimulus was $98 \mathrm{mc} / \mathrm{sec} / \mathrm{cm}^{2}$, which the majority of Ss described as warm. Each S took part in one control session and in one experimental session. In the control session, pain threshold was assessed in the usual way; that is, the intensity of each successive stimulus was increased by approximately $16 \mathrm{mc} / \mathrm{sec} / \mathrm{cm}^{2}$, since Haslam \& Thomas (1967) have found this interval to be optimal for the assessment of pain thresholds, in the sense of giving the most reliable threshold values. The stimulus intensity was increased in this way until the $S$ reported a small stab of pain at the end of a 3 -sec exposure. In the experimental session an anchor was introduced prior to the assessment of pain threshold: the starting-point stimulus was repeated 30 times. After this, the intensity of each successive stimulus was increased by approximately $16 \mathrm{mc} / \mathrm{sec} / \mathrm{cm}^{2}$. In this way, the last of the anchor stimuli became the first of the scale.
Half of the Ss underwent the experimental session first and the other half underwent the control session first. The experimental and control sessions were separated by at least 3 days.

The instructions geven to Ss before stimulation began were identical for both sessions. Ss were told that the stimulus would probably first be felt as warm, that this warmth would gradually increase and would be felt as hot, and that finally a small stab of pain would be felt at the end of a 3-sec exposure. In addition, they were told that the procedure was not an endurance test and was not intended to discover how much pain they could stand but was an experiment to find out at what stage the stimulus was first felt as painful.

Twenty students, 10 male and 10 female, served as Ss. Their mean age was 21.0 years.

\section{RESULTS}

Table 1 shows the mean pain threshold and range for the experimental and control sessions. A Wilcoxon matched-pairs signed-ranks test (Siegel, 1956) showed that pain thresholds for the experimental session were significantly lower than for the control session ( $p<0.005$, one-tailed). The prediction was thus confirmed.

A Mann.Whitney $U$ test showed that there was no significant difference in pain threshold between those Ss who underwent the control session first and those who underwent the experimental session first, and there was no significant difference between male and female pain thresholds for either session.

Although in the experimental session the first 30 stimuli were of the same intensity, Ss did not report them as feeling the same. Some were reported as warmer and some as cooler. On being questioned after the experiment, Ss made various suggestions as to what procedure was being adopted, e.g., some thought that jnds were being measured, and some thought that the stimulus was being presented in a random fashion, i.e., that sometimes it was of greater intensity and sometimes less.

\section{DISCUSSION}

The results indicate that pain threshold is susceptible to an anchoring effect. The low threshold values found when a small stimulus interval is used in the assessment of heat-pain threshold by the limiting method can, therefore, probably be attributed to this effect. That is, a series of low-intensity stimuli alters a S's frame of reference with the result that his "adaptation level" (Helson, 1947) is lowered. If the pain threshold is considered to be a form of adaptation level (AL), that is, the point which is intermediate between nonpainful heat and fairly intense pain, then a series of low-intensity stimuli can be thought of as exerting an anchoring eîfect on the AL similar to that found in many other cases of psychophysical judgment.

The finding reported above may have implications for other experimental results arising in connection with prolonged periods of stimulation. For example, Frankenhaeuser et al (1966) found that repeated application of suprathreshold electrical stimulation produced a rise in sensation threshold and that the magnitude of the change in threshold increased with increasing stimulus intensity. The authors suggested that the reason for the change in sensitivity was physiological and may have been attributable to the prolonged passage of current through the tissues. However, their results may perhaps be explained in terms of an anchoring effect, especially as one of the characteristics of this effect is that the further an anchor stimulus is removed from the range of stimuli being judged, the greater is the shift in a S's judgment scale in the direction of the anchor. Sensation threshold can be considered as a form of adaptation level, that is, the point which is intermediate between no sensation and definite sensation, and repeated application of suprathreshold stimuli can be thought of as exerting an anchoring effect, in that the judgment scale moves in the direction of the anchor.

In any event, an anchoring effect has been demonstrated for heat-pain threshold, and this discussion is intended (1) to underline the need for carefully controlled conditions when sensory thresholds are being investigated and (2) to point out that experimental procedures which involve prolonged periods of stimulation may yield anomalous results attributable to an altered frame of reference.

\section{REFERENCES}

FRANKENHAELSER, M., MELLIS, I., \& FRÖBERG, J. The effect of electrical stimulation on sensation threshold. Report of the Psychological Laboratory, Lniversity of Stockholm, 1966. No. 207.

HARDY, J. D., WOLFF, H. G., \& GOODELL. H. Pain sensations and reactions. Baltimore: Williams \& Wilkins. 1952.

HASLAM, D. R. The influence of stimulus scale-interval upon the assessment of pain threshold. Quarterly Journal of Experimental Psychology, 1965. 17, 65-68.

HASLAM, D. R. Some anomalies in the study of experimental pain. Quarterly Journal of Experimental Psychology. 1968, 20. 208-211. HASLAM, D. R., \& THONAS, E. A. C. An optimum interval in the assessment of pain threshold. Quarterly Journal of Experimental Psychology, 1967, 19, 54-58.

HELSON, H. Adaptation-level as a frame of reference for prediction of psychophysical data. American Journal of Psychology, 1947. 60. 1-29.

SIEGEL. S. Comparametric statistics for the behavioral sciences. New York: McGraw-Hill. 1956. 